\title{
Current computational transportation science
}

\author{
Stephan Winter ${ }^{1} \cdot \mathrm{Xin}_{\mathrm{Chen}^{2}} \cdot \mathrm{Bo} \mathrm{Xu}^{2}$
}

Published online: 15 March 2016

C) Springer Science+Business Media New York 2016

\begin{abstract}
This special issue collects current contributions to Computational Transportation Science, the science behind intelligent transportation systems. In our editorial we reflect on the fundamental nature of this science, and what it means to develop the knowledge for intelligent transportation systems.
\end{abstract}

Keywords Computational transportation science $\cdot$ CTS $\cdot$ Intelligent transportation systems · ITS

\section{Approaching computational transportation science}

Workshops and seminars on Computational Transportation Science, or CTS, have become a fix point over the past few years. And yet the term has not become a common name; it may still require explanation. Computational transportation science has been characterized to be the science behind intelligent transportation systems or ITS [5, 16]. Hence, the far more common term ITS serves us here to make an argument. On first notice, ITS puts the emphasis on systems rather than on science, a disambiguation also common between information systems and information science, probably reminding the readership of this journal of the formation of a geographic inforamtion science on the shoulders of geographic information systems some 25 years ago [7]. A number of papers since then have searched for the core foundations of a geographic information science, and in line with that older debate there would be more to say about the foundations of a computational transportation science. However, this is not the aspect we want to develop in this editorial.

Stephan Winter

winter@unimelb.edu.au

1 Department of Infrastructure Engineering, The University of Melbourne, Parkville, Australia

2 HERE, USA 
Instead, being concerned with computational methods rather than the tangible infrastructure of systems, the sensors and (the tangible bits of) the information and communication technology, it is the term intelligent that stands out for us. It invokes Weiser's vision of the computer disappearing in the environment [13], and then, of course, it requires defining what intelligence actually is. Turing's view on intelligence was one of interaction: The intelligent machine interacts with a person in a way such that the person can no longer distinguish whether it deals with another person or a machine [12]. Turing's suggestion of this test as criterion for (artificial) intelligence reverberates until today [3, 4]. However, in Turing's test it is a person who judges, or an observer of the person, on the premise that persons are intelligent beings. So, can we think of persons judging whether a transportation system is intelligent?

Let us apply this notion to transportation systems and try an interesting mapping: If we consider transport systems as machines of certain observable behaviors, interacting with people, then the system can be called intelligent if these people do no longer recognize whether the system is controlled by people or by machines. For example, for a human car driver the autonomous car in front should 'behave' as if it were driven by a person, and for a pedestrian attending to cross a street the approaching car should interact as if there were eye-contact with a human driver. The research community, although prominently having a human-computer interaction agenda, has not yet defined such a Turing test. The discipline of artificial intelligence would call it a restricted Turing test $[6,10,17]$ since the conversational context of the interaction would be limited to behavior in traffic.

By definition such an approach excludes machine-machine and machine-environment interaction. There is no way to call this interaction intelligent as long as it is not considered from the perspective of a human observer. Thus, technological feats such as the connected vehicle $[9,11]$ are not per se delivering intelligent transportations systems, although they are the cornerstones of current ITS hypes. Telematics achieving connected vehicles is all about behavior: It enables in vehicle-to-vehicle communication and coordination (V2V), facilitating for example early braking systems or vehicle platooning, and also vehicle-toinfrastructure communication and coordination (V2I), facilitating for example electronic toll collection or cooperative traffic light controlling. However, they are not interacting with people and thus not testable to be intelligent.

Only where telematics includes people, facilitated by vehicle-to-anything communication (V2X or Car-to-X), some avenues open for a restricted Turing test - which, again, has not been formulated yet. In contrast the autonomous vehicle mentioned above, the connected vehicle allows at least theoretically other forms of interaction with people: Interaction that includes coordination and collaboration. If only vehicles and people would speak the same language, that is. Thus, a vehicle is needed that not only interprets a person's behavior or language (the autonomous vehicle), but also responds by talking.

Since these interactions between persons and autonomous or connected vehicles are time-critical they have to happen in spoken common language. Neither text-based interfaces nor structured forms of dialog via menus will have the flexibility to "cooperate like another person' in situations of everyday decision making, let alone decision making in stressful or threatening situations. The only other acceptable mode of interaction is embodied behavior: Movement and gestures. The above example aims at the vehicles' movement behavior, but could a vehicle also 'gesture'?

Interaction via common language is a particular challenge for this domain of transport. A restricted conversation will address space and time, or where and when, within the context (coverage) of a particular transport system. Despite all the progress of webmapping systems, 
car navigation systems and even general search about space and time, the spatial domain still has its hard challenges.

One is the flexibility with which people use prepositions: While reportedly four out of the ten most frequent words in the English language are prepositions (of, to, in, for) [1], each of them may be bearing spatial meaning, the same authors also note: "Despite their frequency, however, they are notoriously difficult to master, even for humans" (l.c., p. 119), and: "On the one hand they have been championed as being vital to precise language understanding $[\ldots]$, and on the other they have been ignored on the grounds of being syntactically promiscuous and semantically vacuous, and relegated to the ignominious rank of 'stop word" " (l.c., p. 119).

The ensuing challenge is the lack of accurate, computational models interpreting (spatial) prepositions appropriatly. For example, whether the train station is near the city centre depends solely on the conversational context, and whether at the train station means near, or entering, or inside is also a matter of context, but also likely to be left open by the speaker as the distinctions may be felt irrelevant in that context. So far, artificial intelligence dealing with qualitative spatial reasoning and representation [2] suggests formal models for selected categories of qualitative spatial relationships (topological, cardinal directions, relative directions, and distance), which suit well for reasoning, but which do not easily permit mapping flexible prepositions to these formally defined relationships.

Another challenge the use of locative references in common language. These references can be given in a large variety of forms even for the same location, for example nominally (toponyms), by vernacular (place names unknown by the system), descriptive (types of a place, properties of a place), or by activities ("home"), not to mention by different granularities. Traditional toponym resolution gets quickly stuck. In addition, these references to places manage with conceptual objects that are vague and varying with context [15].

These three challenges for communication between transport systems and people are surprisingly complex for both parties involved in the conversation. Only on the surface they appear to be challenges for natural language understanding and natural language generation. Hidden from the person involved are the challenges for the system to operate and react to this interaction. So, when a car navigation system recommends: "Turn right at the next intersection", the persons in the driver's seat have to match this information with their own perception and conceptualization of the environment. It is neither formally agreed what a right-turn is, nor what the concept of an intersection is. Vice versa, when a driver requests: "Bring me to the café opposite the train station" the system has to address which train station the driver is referring to (potentially out of the conversational context), what a café is [8], which of the candidates in the database can be seen as located opposite of the train station, and what bringing to means (the front door, a nearby parking lot, or a recommendation to use the bus because of a lack of (cheap) parking nearby, as any reasonable person would do). - While these examples are still some sort of traditional (although unsolved), the challenges will only increase in future applications: Which language will truck drivers use to interact with their vehicles travelling in a platoon? What is an appropriate and safe way for cooperation between human driver and vehicle? How will they determine who is responsible for what? How can a system of systems interact in supporting the mobility of people ("City, I have to be at work in 20 minutes")?

At this stage it should have become clear that computational transportation science is about the fundamental challenges for intelligent transportation systems. One last question, however, is worth addressing: Why should transportation systems be 'intelligent' at all? Wouldn't be systems that we might call here smart - without further definition, but not 
necessarily intelligent - be sufficient? Or perhaps even be superior? What if the system knows better than us [14]? French already remarked: "[F]or example, do we really need to build computers that make spelling mistakes or occasionally add numbers incorrectly, as in Turings original article, in order to fool people into thinking they are human?" [4, p. 74]. A simple imitation game has plenty of anecdotes about people's limitations of providing directions to choose from, and clearly imiation, or shallowly fooling people into being human, is not a goal for intelligent transportation system. So, as French continues, "building machines that would never pass a Turing Test, but that can interact with humans in a highly meaningful, informative way" (l.c., p. 74) is the way forward. Unfortunately, however, French's argument has lost the clear definition of intelligence by Turing. We argue here instead for a proper understanding of Turing's test: A restriction of the Turing test to the domain of mobility, limited to the intuitive and meaningful interaction of people and systems that are supporting safe, transparent and efficient mobility.

Understood this way, a Turing test helps to carve out the basic questions of a computational transportation science. A Turing test is a constant reminder that there is no transport system without people involved. It also would be the first benchmark in the domain of intelligent transportation systems allowing to compare systems with each other. And finally: Yes, the system can provide smart solutions to me - I accept that there are also persons out there smarter than I am.

\section{The papers of this special issue}

The article by Feihu Zhang, Gereon Hinz, Dhiraj Gulati, Daniel Clarke, and Alois Knoll, titled "Cooperative Vehicle-Infrastructure Localization based on the Symmetric Measurement Equation Filter", focuses on high accuracy localization which is an important problem for safe autonomous driving. The authors consider a traffic scenario in which vehicles are equipped with internal sensors for self-localization, and there are external sensors from the infrastructure for vehicle localization. In such a scenario vehicle-infrastructure communication can be used to improve the localization accuracy. However, as the number of the tracked vehicles increases, associating measurement data with the correct vehicle raises a significant computational challenge. The authors propose a solution that utilizes the symmetric measurement equation filter (SME) for cooperative localization to avoid measurement-totarget associations. The main idea is to define a symmetrical transformation which maps measurements to a homogeneous function, thereby effectively dealing with several difficulties in vehicle-infrastructure scenarios including data association, bandwidth limitations and registration/configuration of the external sensor. The proposed method is evaluated by simulations and the results show that the SME filter produces higher localization accuracy than two existing methods namely the Kalman filter and the Probability Hypothesis Density (PHD) filter, but with greatly reduced computational and communicational cost.

Bing Zhang, Goce Trajcevski and Liu Liu's article titled "Towards Fusing Uncertain Location Data From Heterogeneous Sources" studies the problem of combining the uncertain location data obtained from GPS devices and roadside sensors. The authors propose a novel model called Fused Bead (FB) to capture the whereabouts of moving objects in time in this setting. The FB model extends from the existing bead model which is based solely on GPS locations. The authors further develop algorithms for answering traditional spatiotemporal range queries and a special variant pertaining to objects locations with respect to lanes on road segments. These algorithms facilitate the processing of so-called lane-crossing 
queries, e.g., "Retrieve all the vehicles which have had more than $90 \%$ probability of crossing the lane in road segment RS1 when driving less than $50 \mathrm{~km} / \mathrm{h}$ and carrying less than $80 \%$ of the maximum load". The answers to such queries play an important role in applications related to efficient traffic management for the purpose of regulating the regime of traffic lights. The authors evaluate the benefits of the proposed approach by experiments on both real and synthetic data. The experiments show that the proposed method may reduce false positives by up to $26 \%$ for the real data, and by up to $40 \%$ for the synthetic dataset, when compared with the traditional bead uncertainty models.

The article by Paul Weiser, Simon Scheider, Dominik Bucher, Peter Kiefer, Martin Raubal, titled "Towards Sustainable Mobility Behavior: Research Challenges for LocationAware Information and Communication Technology", investigates how location-aware information and communication technology (ICT) can enable novel interactive and participatory approaches that help people in becoming more sustainable. The authors discuss the various aspects of this issue from a technological and cognitive engineering perspective, based on an overview of the main information processes that may in influence mobility behavior. They provide a systematic survey of research challenges, the corresponding state-of-the-art, and future research opportunities of location aware ICT targeted at making people's behavior more sustainable. The survey starts with an analysis of the information requirements for some established behavior to be changed, taking into account both the analysis and planning aspects of sustainable activity alternatives. The survey then identifies open research questions via a literature review on the state-of-the-art research, ranging from goal planning and activity detection, over activity scoring, to the effective communication of sustainable alternatives. As a conclusion the survey suggests a research agenda that aims at tackling the identified open challenges.

Acknowledgments S. Winter's work is supported by the Australian Research Council (LP120200130).

\section{References}

1. Baldwin T, Kordoni V, Villavicencio A (2009) Prepositions in applications: a survey and introduction to the special issue. Comput Linguis 35(2):119-149

2. Cohn AG, Renz J (2008) Qualitative spatial representation and reasoning, chap. 13, pp 551-596. Elsevier

3. French RM (2000) The turing test: the first fifty years. Trends Cognit Sci 4(3):115-121

4. French RM (2012) Moving beyond the turing test. Commun ACM 55(12):74-77

5. Geers G Some research questions for computational transportation science. In: 5th annual international conference on mobile and ubiquitous systems. Institute for Computer Sciences, Social-Informatics and Telecommunications Engineering

6. Geman D, Geman S, Hallonquist N, Younes L (2015) Visual Turing test for computer vision systems. Proc Nat Acad Sci 112(12):3618-3623. doi:10.1073/pnas.1422953112. http://www.pnas.org/content/ 112/12/3618.abstract

7. Goodchild MF (1992) Geographical information science. Int J Geograph Inf Syst 6(1):31-45

8. Janowicz K, Raubal M (2007) Affordance-based similarity measurement for entity types, lecture notes in computer science, vol 4736. Springer, Berlin, pp 133-151

9. Ring T (2015) Connected cars: the next target for hackers. Netw Secur 2015(11):11-16. doi:10.1016/S1353-4858(15)30100-8

10. Shieber SM (1994) Lessons from a restricted Turing test. Commun ACM 37(6):70-78

11. Swan M (2015) Connected car: quantified self becomes quantified car. J Sensor Actuator Netw 4(1):229. doi: $10.3390 /$ jsan 4010002

12. Turing AM (1950) Computing machinery and intelligence. Mind 59(236):433-460

13. Weiser M (1991) The computer for the twenty-first century. Scie Amer 9:94-104 
14. Winter $\mathrm{S}$ (2012) Intelligent geospatial systems: how far is HAL-NAV? Geospatial World 11:46-48

15. Winter S, Freksa C (2012) Approaching the notion of place by contrast. J Spatial Inf Sci 2012(5):31-50

16. Winter S, Sester M, Wolfson O, Geers G (2011) Towards a computational transportation science. J Spatial Inf Sci 1(2):119-126. http://josis.org/index.php/josis/article/view/39/40

17. Winter S, Wu Y (2008) Towards a conceptual model of talking to a route planner, lecture notes in computer science, vol 5373. Springer, Berlin, pp 107-123

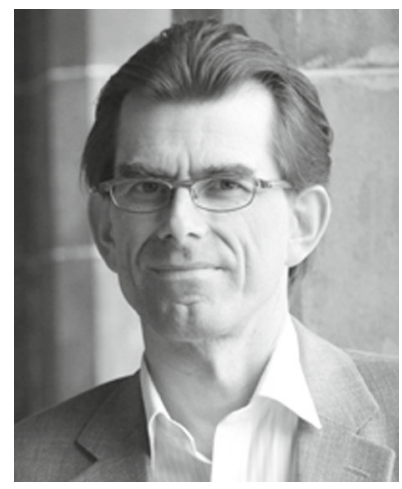

Stephan Winter is Professor in Spatial Information Science at the Department of Infrastructure Engineering, The University of Melbourne. He holds a PhD (Dr.-Ing., 1997) from the University of Bonn, and a habilitation from the Technical University Vienna (2001). Within spatial information science Stephan Winter is specializing on human wayfinding and navigation, with a vision of developing intelligent spatial machines. He has contributed to topics such as spatial human-computer interaction, network analysis, routing heuristics, and collaborative transportation and evacuation.

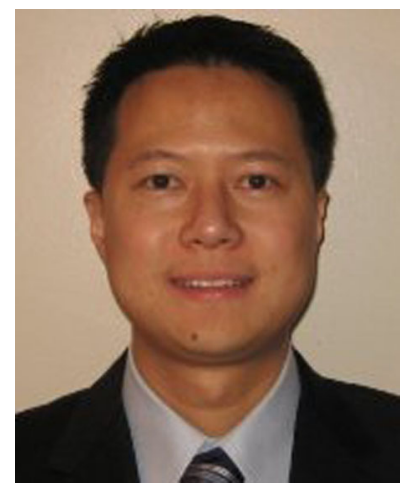

Xin Chen is a Senior Engineering Manager and Research Scientist at HERE leading a team completing pioneering work to achieve the automation of next generation map creation using computer vision and machine learning technologies. Xin is also an adjunct professor at Northwestern University and Illinois Institute of Technology. He has numerous patents and academic publications. Xin obtained his Ph.D. in Computer Science and Engineering from the University of Notre Dame. 


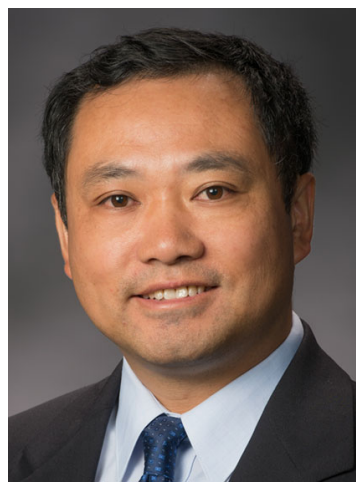

Bo Xu is a Senior Researcher at HERE. Before joining HERE, he conducted research in Argonne National Laboratory and University of Illinois at Chicago. His research interests include computational transportation science, urban computing, and spatio-temporal databases. He has published over 80 scholarly papers on subjects such as traffic delay analysis, agent based traffic simulation, mobile peer-to-peer databases, and moving objects databases. Bo received his Ph.D. in Computer Science from University of Illinois at Chicago and from University of Electronic Science and Technology of China, respectively. 